\title{
Graph of Words Embedding for Molecular Structure-Activity Relationship Analysis
}

\author{
Jaume Gibert ${ }^{1}$, Ernest Valveny ${ }^{1}$, and Horst Bunke ${ }^{2}$ \\ 1 Computer Vision Center, Universitat Autònoma de Barcelona \\ Edifici O Campus UAB, 08193 Bellaterra, Spain \\ \{jgibert, ernest\}@cvc.uab.es \\ 2 Institute for Computer Science and Applied Mathematics, University of Bern, \\ Neubrückstrasse 10, CH-3012 Bern, Switzerland \\ bunke@iam.unibe.ch
}

\begin{abstract}
Structure-Activity relationship analysis aims at discovering chemical activity of molecular compounds based on their structure. In this article we make use of a particular graph representation of molecules and propose a new graph embedding procedure to solve the problem of structure-activity relationship analysis. The embedding is essentially an arrangement of a molecule in the form of a vector by considering frequencies of appearing atoms and frequencies of covalent bonds between them. Results on two benchmark databases show the effectiveness of the proposed technique in terms of recognition accuracy while avoiding high operational costs in the transformation.
\end{abstract}

\section{Introduction}

Biological properties of molecules, such as chemical reactivity, mutagenicity or anti-cancer activity, are presumed to be correlated with the inherent molecular structure. Such assumption is what lies behind the Structure-Activity Relationship (SAR) analysis, where activity is any of the biological responses molecules might show off. Chemoinformatics has extensively dealt with this problem, usually by representing molecules in the form of labelled undirected graphs [8], describing the 2D structure of the chemical compounds. However, working with this molecular representation leads to the usual problem one encounters when dealing with graph-based representations, namely, the high computational complexity of the analysis and comparison of graphs, also known as graph matching 1 .

Common sub- and super-graphs between pairs of graphs may be used to define graph similarity for the problem of graph matching [46]. Also graph edit distance is a powerful tool that defines similarity between graphs by the amount of distortion that is needed to transform one graph into another [5]2. Even though there exist suboptimal solutions to these problems, they still suffer from high complexity.

In the last years, two new lines of research have been opened which allow the classical statistical machine learning methodology being applied to structural

I. Bloch and R.M. Cesar, Jr. (Eds.): CIARP 2010, LNCS 6419, pp. 30-37, 2010.

(C) Springer-Verlag Berlin Heidelberg 2010 
pattern recognition problems. Graph embedding, which associates a feature vector to each graph, and graph kernels, which define a kernel function between instances of graphs, are emerging and promising fields that aim at exploiting the benefits of both the representational power of graphs and the wealth of algorithmic tools of statistical machine learning.

The new method we present in this paper aims at performing the SAR analysis by embedding graph molecules into feature spaces. The next section sets out the details of the proposed procedure. Then, Section 3 presents a comparison of our methodology with different state-of-the art techniques using two different databases. Finally, Section 4 concludes the article.

\section{Embedding of Molecular Graphs}

The molecules embedding procedure we propose here associates a feature vector to each molecule, initially represented by a graph. It is originally based on a well-known approach to document and image classification [11, which is also called bag of words technique. The main idea behind it can be summarized as follows. By taking a glance at a document we can just describe its layout and the distribution of the paragraphs, but we can barely say to which class this document belongs. However, by taking a more careful look we may notice the existence of keywords that can give us some hints on the document topic. For instance, if we detect that in the document the words brain, cell, disease, doctor, etc. frequently appear, we can consider such a document as a medical one.

Technically, we need to provide a set of keywords, also known as vocabulary, and then just count the frequency of appearance of each keyword in the document. This results in a histogram of keywords appearing in the document that is used as the document representation. The histograms can be used as feature vectors to feed any machine capable to learn the document classes and perform the categorization task.

In this paper, we aim at defining a similar procedure with molecules. The nodes of a molecule graph are attributed with a chemical element. By letting the set of chemical elements be our vocabulary -in the sense it has been explained above- we can create a histogram of atoms appearing in each molecule. Yet, the fact that atoms in the molecule are linked by covalent bonds leads to an even richer description. To obtain such an enrichment, we need to transform the molecule graph into a new graph we shall call the graph of words. From this new graph, we will obtain the feature vector by taking into account node and edge attributes. The next sections formally define the embedding of molecules in vector spaces and two different ways of handling edge attributes.

\subsection{Formal Definitions}

The graph of words. Assume a set of molecules $M=\left\{g_{1}, \ldots, g_{n}\right\}$ is given. Each molecule is represented by a graph $g_{i}=\left(V_{i}, E_{i}, \mu_{i}, \nu_{i}\right)$, where $V_{i}$ is the set of nodes, $E_{i} \subseteq V_{i} \times V_{i}$ the set of edges, $\mu_{i}$ is the function assigning atomic 


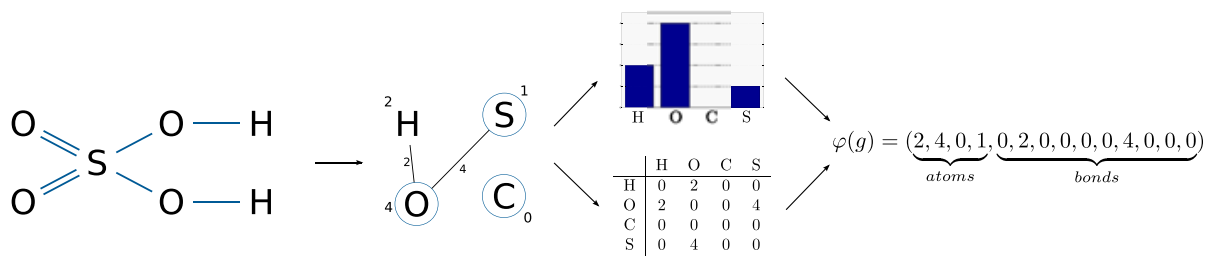

Fig. 1. Embedding of the Sulphuric Acid molecule using the ordered vocabulary $\mathcal{V}=\{H, O, C, S\}$. The first transition shows the graph of words construction for the molecule at the left. Following, the histogram of appearing atoms/words and the adjacency matrix of the graph of words are shown. Finally, the vector representation is the concatenation of both the histogram of atoms and the right upper diagonal part of the adjacency matrix.

elements to the nodes $v \in V_{i}$ and $\nu_{i}$ assigns to each edge $(u, v) \in E_{i}$ the kind of covalent bond that links the atoms.

Following the idea explained above, we define the vocabulary $\mathcal{V}$ by the set of existing atomic elements in any of the molecules of $M$. In the histogram representation we would just count the frequency of each node label in a graph, but since we want to use the bond information as well we transform each molecule $g=(V, E, \mu, \nu)$ into another graph $g^{\prime}=\left(V^{\prime}, E^{\prime}, \mu^{\prime}, \nu^{\prime}\right)$ where

$-V^{\prime}=\mathcal{V}$,

- $E^{\prime}$ is defined by: $\left(w, w^{\prime}\right) \in E^{\prime} \Leftrightarrow$ there exists $(u, v) \in E$ such that $\mu(u)=w$ and $\mu(v)=w^{\prime}$,

- $\mu^{\prime}$ is the mapping $\mu^{\prime}(w)=|\{v \in V \mid \mu(v)=w\}|$,

- and $\nu^{\prime}$ is the edge labelling function defined by $\nu^{\prime}\left(w, w^{\prime}\right)=\left|\left\{(u, v) \in E \mid \mu(u)=w, \mu(v)=w^{\prime}\right\}\right|$.

In plain words, we represent a molecule graph by another graph whose nodes are the node vocabulary $\mathcal{V}$; note that each atom appears just once. We call this new graph the graph of words, in analogy to the bag of words representation. We label each node of the new graph by the frequency of occurrence of the corresponding atom. Note that this definition allows for an atom to be labelled with the zero attribute. Edges of the graph of words are those links between nodes that also occur in the molecule. For instance, if there is a bond between two atoms $C$ and $H$ in the molecule then we link the words $C$ and $H$ in the new representation. The label of such an edge will tell us how many times the corresponding edge occurs. Note that zero attributed edges are allowed and represent the fact that two atoms are not linked in the molecule.

By this procedure, we do not only have all information included in the histogram of words, but also how the words/atoms are structurally related in the molecule. The first transition step in Figure 1 depicts an example of this representation for a simple vocabulary of just four atoms.

Vector representation. Based on the graph of words representation it is straightforward to assign a feature vector to every molecule in $M$. Since we 
want to keep both the information about the atoms and the bonds, we split the graph of words into a histogram of atoms and the adjacency matrix (second transition in Figure 1). Let $N$ be the number of atoms in the vocabulary. The histogram of atoms for all graphs in $M$ can be formally written as a mapping $\phi_{a}: M \rightarrow \mathbb{R}^{N}$, where

$$
\phi_{a}(g)=\left(\mu^{\prime}\left(w_{1}\right), \ldots, \mu^{\prime}\left(w_{N}\right)\right) .
$$

The adjacency matrix of the graph of words is an $N \times N$ symmetric matrix $A=\left(a_{i j}\right)$, where each entry $a_{i j}$ describes the frequency of the relation between the $i$ th and $j$ th atoms, this is, $a_{i j}=\nu^{\prime}\left(w_{i}, w_{j}\right)$. The fact that we control the vocabulary, makes this matrix easily sortable since, for every molecule, each entry (feature) of the matrix is describing the same information. Also, due to the symmetry of the matrix we can just consider the diagonal and the upper part of it. Therefore, we arrange the graph of words adjacency matrix as $\phi_{b}: M \rightarrow \mathbb{R}^{p}$, where

$$
\phi_{b}(g)=\left(a_{11}, \ldots, a_{i j}, \ldots, a_{N N}\right), \quad \forall i \leq j
$$

and where $p=\left(N^{2}+N\right) / 2$. Finally, we can just concatenate both the atomic histogram information and the structural relations of atoms in a single vector as follows: $\varphi: M \rightarrow \mathbb{R}^{N+p}$, where

$$
\varphi(g)=\left(\phi_{a}(g), \phi_{b}(g)\right)
$$

The last transition of Figure 1 shows the final vector representation of the molecule on the left.

\subsection{Edge Attributes Handling}

The reader may have noticed that in the construction defined above the edge attributes of the molecules have been ignored. That is, there has been no attention paid on whether the atomic bond is either single or double. This representation is what we call $R_{1}$ or all-in-one, since the edges are all stored in one adjacency matrix. As an alternative, to consider the type of atomic bonds, we also define the $R_{2}$ or separated representation: based on the same construction, we may consider an adjacency matrix for each type of edge label separately and then concatenate all matrices in a single final vector.

For instance, in the example of Figure 11, the $R_{1}$ representation is the one depicted since edge attributes are neglected. For the second representation $R_{2}$ we should take the matrices

$$
A_{s}=\left(\begin{array}{llll}
0 & 2 & 0 & 0 \\
2 & 0 & 0 & 2 \\
0 & 0 & 0 & 0 \\
0 & 2 & 0 & 0
\end{array}\right) A_{d}=\left(\begin{array}{llll}
0 & 0 & 0 & 0 \\
0 & 0 & 0 & 2 \\
0 & 0 & 0 & 0 \\
0 & 2 & 0 & 0
\end{array}\right)
$$

where $A_{s}$ is representing the single bonds and $A_{d}$ the double bonds. With this procedure, even though the final vector representation under $R_{2}$ will be larger 
and more sparse than under $R_{1}$, it will still keep information of the atomic bonds of the original molecule. In the experiments section, we will explore the two alternatives and discuss which one of our embedding procedures is more suitable.

\subsection{Remarks}

After the formal definition of our embedding has been given, we would like to point out its two strong points. On the one hand, the feature vector we assign to each molecule is storing both statistical and structural information. The first part of the vector $\phi_{a}$ is counting occurrences of atomic elements in the molecule, while the second part $\phi_{b}$ is describing the structure of these elements in the molecule. On the other hand, in contrast to most of the existing graph embedding techniques and feature extraction algorithms for graphs, our approach is very inexpensive in terms of algorithmic complexity. In fact, for a graph $g$ with $n$ nodes, only $\mathcal{O}\left(n^{2}\right)$ operations are required to transform $g$ into $\varphi(g)$. This is in sharp contrast with other embedding procedures, for example [3], which are exponential in $n$.

\section{$3 \quad$ Experiments}

\subsection{Databases}

We applied our molecular embedding based on the graph of words representation to two different benchmark datasets of molecules. The Predictive Toxicology Challenge (PTC) database [7] is the result of a pharmaceutical experiment in which several molecular compounds are tested in four types of animals: Male Mouse (MM), Female Mouse (FM), Male Rat (MR) and Female Rat (FR). Each compound is assigned to either the positive or the negative class according to its carcinogenicity activity. This results in four two-class supervised classification problems in which the activity of the compounds for a specific animal should be discovered. The second set, the MUTAG database [13, is a set of molecular compounds tested for mutagenicity activity on Salmonella typhimurium. Again, the problem is a binary supervised classification task consisting of the determination of the activity of the molecules.

In Table 1 we show some statistics for each molecular database. In particular, we give the total number of molecules in every dataset (\#TOTAL); how many of them are positive for carcinogenicity/mutagenicity (\#POSITIVE); how many are negative (\#NEGATIVE); the maximum order (number of nodes) in each dataset (MAX $|G|$ ) and the average order (AVG $|G|$ ); the number of different node attributes in the dataset $\left(|\Sigma|_{v}\right)$, which is the number of atoms/words in the vocabularies we use for the proposed embedding; and the number of different types of atomic bonds in the molecules $\left(|\Sigma|_{e}\right)$, which would be the number of adjacency matrices to consider in the $R_{2}$ representation of the graph of words embedding. 
Table 1. PTC and MUTAG Datasets Statistics

\begin{tabular}{lccccc}
\hline & MM & FM & MR & FR & MUTAG \\
\hline \#TOTAL & 336 & 349 & 344 & 351 & 188 \\
\#POSITIVE & 129 & 143 & 152 & 121 & 125 \\
\#NEGATIVE & 207 & 206 & 192 & 230 & 63 \\
MAX $|G|$ & 109 & 109 & 109 & 109 & 28 \\
AVG $|G|$ & 25.0 & 25.2 & 25.6 & 26.1 & 18.0 \\
$|\Sigma|_{v}$ & 21 & 19 & 19 & 20 & 8 \\
$|\Sigma|_{e}$ & 4 & 4 & 4 & 4 & 4 \\
\hline
\end{tabular}

\subsection{Reference Systems}

In order to compare our results, we have selected two articles that report about molecular classification on the same datasets. In [9], a marginalized graph kernel between labelled graphs is proposed and compared to the Pattern Discovery algorithm [10]. The marginalized graph kernel is defined as the expectation of the joint kernel between labelled paths, where such paths are defined under a first-order Markov random model. The Pattern Discovery algorithm assigns each molecule to a feature vector where each attribute is counting the number of occurrences of a certain label path in the molecule. On the other hand, in 14, the authors exploit the fact that molecules can either be represented by $1 \mathrm{D}$ structures (SMILE strings), 2D structures (graphs), or 3D representations where spatial coordinates of the atoms are taken into account. Based on these facts, several kernels are defined for each representation. We will report here just the best ones for each case.

Even though there exist optimal and fast computational procedures for these approaches, they all suffer from higher complexity than ours since they are based on searching substructures in the graphs.

\subsection{Results}

Leave-one-out validation consists in testing every element in the dataset with the learnt model from the rest of existing available patterns. The accuracy rate of the whole system is the number of correctly classified patterns out of the total number in the dataset. In this section we detail the classification rate for the databases using leave-one-out validation and Support Vector Machines [12] as the learning machine.

In Figure 2 we see how the $R_{1}$ representation outperforms in all cases the $R_{2}$ representation, which takes into account the molecular bond types. This situation seems to be due to the sparsity of the $R_{2}$ representation. In Table 1 we can see that there are four different types of molecular bonds in the datasets. However, two of these four types barely appear as an edge attribute and so the proportion between these types and the other two is really low. Such circumstance creates adjacency matrices of the graph of words with almost all entries being zero; thus, the resulting vector representation consists of many zero values and just a few 


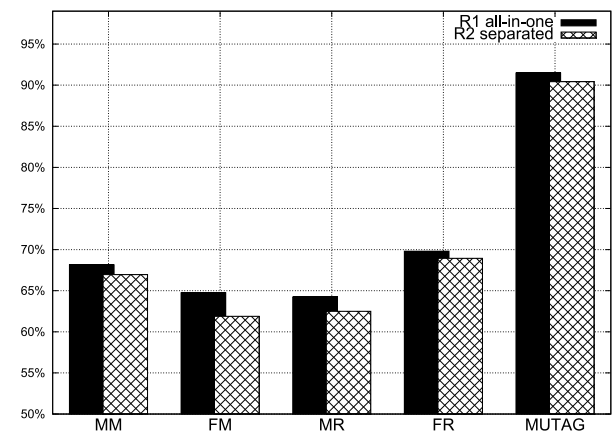

Fig. 2. Comparison of the accuracy rates obtained for all databases using the two proposed configurations $R_{1}$ and $R_{2}$ of the molecular embedding

Table 2. Comparison of leave-one-out results for different datasets using several methods. Accuracy rates in \%. The best method on each dataset in printed bold face.

\begin{tabular}{lccccc}
\hline & MM & FM & MR & FR & MUTAG \\
\hline Pattern Discovery [9] & 61.0 & 61.0 & 62.8 & 66.7 & 89.9 \\
Walk kernel 9] & 64.3 & 63.4 & 58.4 & 66.1 & 85.1 \\
Best 1D kernel [14] & 66.4 & 63.0 & 57.6 & 67.0 & 85.6 \\
Best 2D kernel [14] & 66.4 & 64.5 & $\mathbf{6 5 . 7}$ & 66.9 & 87.8 \\
Best 3D kernel [14] & 59.8 & 61.0 & 60.8 & 64.4 & 81.9 \\
\hline Proposed $\left(\varphi, R_{1}\right)$ & $\mathbf{6 8 . 1 5}$ & $\mathbf{6 4 . 7 5}$ & 64.24 & $\mathbf{6 9 . 8 0}$ & $\mathbf{9 1 . 4 8}$ \\
Proposed $\left(\varphi, R_{2}\right)$ & 66.96 & 61.89 & 62.50 & 68.94 & 90.42 \\
\hline
\end{tabular}

non-zero ones. This is a plausible explanation that the SVM is capable to better learn the positive and negative classes for the $R_{1}$ representation than for the $R_{2}$.

In Table 2 a comparison between the reference methods and our two configurations of the graph of words embedding is shown for all databases. In four out of the five considered two-class classification problems it is actually superior to the other techniques. Moreover, since the proposed embedding has a low computational complexity, the whole system is worth considering.

\section{Conclusions}

In this paper, we have introduced a new embedding procedure of graph molecular compounds for the problem of structure-activity relationship analysis. The embedding is based on the idea of bag of words for document classification in which a document is represented by a histogram of appearing keywords in the text. Our approach takes the atomic elements appearing in the database and proceeds similarly to the bag of words methods. In order to take profit of the atomic bonds in molecules, we transform the molecule graph into the graph of words representation, in which not only the node histogram information is stored 
but also how the atomic vocabulary is structurally related. As the underlying vocabulary is uniquely given, the arrangement of the new graph representation into a vector is a straightforward step, resulting in a feature vector for each molecular compound.

Results with two different configurations of the embedding have been shown, as well as a comparison with several state-of-the-art molecules classification techniques. The results reveal that the proposed procedure is at the same level as such techniques, while avoiding the computational complexity problems typically encountered in graph-based problems.

\section{References}

1. Conte, D., Foggia, P., Sansone, C., Vento, M.: Thirty years of graph matching in pattern recognition. International Journal of Pattern Recognition and Artificial Intelligence 18(3), 265-298 (2004)

2. Gao, X., Xiao, B., Tao, D., Li, X.: A survey of graph edit distance. Pattern Analysis and Applications 13(1), 113-129 (2010)

3. Riesen, K., Bunke, H.: Graph Classification and Clustering Based on Vector Space Embedding. World Scientific, Singapore (2010)

4. Bunke, H., Shearer, K.: A graph distance metric based on the maximal common subgraph. Pattern Recognition Letters 19(3), 13-25 (1998)

5. Bunke, H., Allerman, G.: Inexact graph matching for structural pattern recognition. Pattern Recognition Letters 1, 245-253 (1983)

6. Fernandez, M.L., Valiente, G.: A graph distance metric combining maximum common subgraph and minimum common supergraph. Pattern Recognition Letter 22(6-7), 753-758 (2001)

7. Helma, C., King, R., Kramer, S., Srinivasan, A.: The Predictive Toxicology Challenge 2000-2001. Bioinformatics 17, 107-108 (2001)

8. Helma, C., Kramer, T., Kramer, S., De Raedt, L.: Data Mining and Machine Learning Techniques for the Identification of Mutagenicity Inducing Substructures and Structure-Activity Relationship of Noncongeneric Compounds. Journal of Chemical Information and Computer Sciences 44(4), 1402-1411 (2004)

9. Kashima, H., Tsuda, K., Inokuchi, A.: Marginalized Kernels Between Labeled Graphs. In: Proceedings of the 20th International Conference on Machine Learning, pp. 321-328. AAAI Press, Menlo Park (2003)

10. Kramer, S., De Raedt, L.: Feature construction with version spaces for biochemical application. In: Proceeding of the 18th International Conference on Machine Learning, pp. 258-265 (2001)

11. Lewis, D.: Naive (Bayes) at Forty: The Independence Assumption in Information Retrieval. In: Proceedings of the 10th European Conference on Machine Learning, vol. (1398), pp. 4-15 (1998)

12. Schölkopf, B., Smola, A.J.: Learning with Kernels: Support Vector Machines, Regularization, Optimization, and Beyond. MIT Press, Cambridge (2001)

13. Srinivasan, A., Muggleton, S., King, R.D., Sternberg, M.: Theories for mutagenicity: a study of first-order and feature based induction. Artificial Intelligence 85, 277-299 (1996)

14. Swamidass, S.J., Chen, J., Bruand, J., Phung, P., Ralaivola, L., Baldi, P.: Kernels for small molecules and the prediction of mutagenicity, toxicity and anti-cancer activity. Bioinformatics 21, 359-368 (2005) 